\title{
Phenotypic detection of Extended Spectrum Beta lactamase production from E. coli and K. pneumoniae in urinary samples among children
}

\author{
Kalyan Subedi ${ }^{1}$, Farisna Karki ${ }^{1}$, Sanju Lama ${ }^{1}$, Agya Pandey ${ }^{1}$, Unita Dahal ${ }^{1}$, Rabin Paudyal ${ }^{1}$
}

${ }^{1}$ Department of Microbiology, Kathmandu College of Science and Technology, Kamalpokhari, Kathmandu, Nepal

Corresponding author: Kalyan Subedi, Department of Microbiology, Kathmandu College of Science and Technology, Kamalpokhari, Kathmandu, Nepal; E-mail-ksubedi@kist.edu.np

\begin{abstract}
Objectives: The main objective of this study was to detect antimicrobial drug resistance (AMR) and Extended Spectrum Betalactamase (ESBL) production phenotypically in E. coli and K. pneumoniae isolated from urines with significant bacteriuria.
\end{abstract}

Methods: This cross-sectional study was carried out in Microbiology laboratory of Kathmandu College of Science and Technology, Kamalpokhari. The urine samples from suspected urinary tract infected cases were collected from both genders of children below 15 years of age from Out and In-patient department of International Children Friendship Hospital, Maharajgunj and those with significant bacteriuria were cultured for isolating the bacterial etiology targeted as E. coli and $K$. pneumoniae. AMR for these two bacteria were tested and detected using Kirby Bauer Disc Diffusion technique. ESBL production was confirmed by Double Disc Synergy test (DDST) and Phenotypic Confirmatory Disc Diffusion Test (PCDDT) after screening for all the isolates showing resistance to third generation cephalosporin namely Cefotaxime and Ceftriaxone according to CLSI instructions.

Results: Out of 388 urine samples processed, 29.89\% (116/388) showed significant bacterial growth. Five (5) different Aerobic Gram Negative bacterial species were detected and identified. E. coli topped the list $(70.68 \%)$ followed by K. pneumoniae (15.52\%), K. oxytoca (8.62\%), Proteus vulgaris $(3.45 \%)$ and Pseudomonas aeruginosa (1.73\%). Among positively screened (44.82\%) beta lactamase producers $(36.2 \%)$ of total isolates were confirmed to produce ESBL. Among ESBL producing isolates, highest susceptibility was seen to Ceftazidime (23.80\%) followed by Cefotaxime $(16.67 \%)$. The ESBL producing isolates were least susceptible to Ceftriaxone (2.38\%). AMR was detected using KirbyBauer Disc diffusion technique. Comparatively less resistance to amikacin and nitrofurantoin (19.1\% and $9.53 \%$ respectively) was seen among ESBL producers. 40 out of the 42 (95.23\%) ESBL producing strains showed susceptibility to the combination drug, piperacillin/tazobactam. The resistance to meropenem was observed to be less $(9.53 \%)$ as compared to that to imepenem $(7.15 \%)$.

Conclusion: This study concluded that there is high prevalence of multidrug resistant uropathogenic clinical strains of E. coli and K. pneumoniae with higher rates of ESBL production. A resistance to the carbepenems is also emerging. Appropriate antimicrobial regimen selection for empirical therapy is thus important for such cases. On managing the empirical antibiotics practice, one can reduce the risk of ESBL producers. There is an essence need of regular routine practice of ESBL detection.

Keywords: AMR, ESBL, E. coli, K. pneumoniae, PCDDT, DDST

\section{INTRODUCTION}

Antimicrobial resistance among bacterial strains causing Urinary tract infections (UTIs), one of the most common bacterial infections in humans both in the

Date of Submission: August 29, 2020

Published Online: December, 2020 community and the hospital settings, is an emerging problem, worldwide. E. coli and K. pneumoniae are the two major pathogens commonly isolated in urine, with $E$. coli being the most prevalent type accounting

Date of Acceptance: October 12, 2020

DOI: https://doi.org/10.3126/tujm.v7i0.33801 
for 75-90\% of UTIs. Usually these infections are treated with a variety of antibiotics, including $\beta$-lactams, $\beta$-lactam/ $\beta$-lactamase inhibitors, flouroquinolones, and carbapenems (Kariuki et al. 2007; Ullah et al. 2009; Hoban et al. 2011 and Briongos-Figuero et al. 2012). However, in recent times, these uropathogens have also developed resistance to commonly prescribed antimicrobial agents; this severely limits the treatment options of an effective therapy.

Primarily, these uropathogens exert their antimicrobial resistance against beta-lactams by producing extended spectrum beta-lactamases (ESBLs) enzymes that confers bacterial resistance to all beta-lactams except carbapenems, cephamycins and clavulanic acid (Coque et al. 2008). It is global matter of concern since infections associated with ESBL producing clinical isolates are found with higher mortality, length of stay, and health care cost and longer antibiotic therapy in comparison to that with non-ESBL producing pathogens (Schwaber and Carmeli 2007). Correct diagnosis and prompt treatment is crucial in order to prevent morbidity and mortality associated with the disease which is further backed up by frequent changing pattern of antimicrobial resistance with development of various resistant mechanisms like drug efflux, reduced uptake and production of hydrolytic enzymes like extended spectrum $\beta$-lactamases (Ghedira et al. 2004). The Infectious Disease Society of America has listed E. coli and Klebsiella species as two out of six pathogens for which new drugs are urgently needed in order to combat their growing resistance (Talbot et al. 2006) The prevalence of ESBL-positive isolates depends on a range of factors including species, geographic locality, hospital/ward, group of patients and type of infection, and large variations have been reported indifferent studies (Livermore et al. 2007).

The different studies from Nepal have indicated a variable rate of ESBL producing bacterial strains in Nepal, where Enterobacteriaceae were found 28\% to $67 \%$ (Hammer et al. 2007). The acquisition and expression of ESBLs enzymes among Enterobactericeae have posed a serious public health problem in developing countries like Nepal that still lacks the facilities for urine culture and antimicrobial susceptibility testing; this clearly leads to missing ESBL isolates in our country. This might be one of the reasons that are creating deaths among urinary tract infected cases in Nepal. Thus, the regular surveillance of the drug resistance among the clinical isolates will be helpful to know the actual burden of the situation, which will help making the necessary policy to reduce the incidence of drug resistance among the bacteria primarily causing UTIs which are being difficult to treat nowadays. This study was carried out to detect antibiotic resistance and phenotypic detection of ESBL among multidrug resistance E. coli and K. pneumoniae to determine the prevalence and antibiotic resistance profile in clinically relevant urine isolates from children.

\section{MATERIALS AND METHODS}

This cross sectional study was carried out at microbiology laboratory of Kathmandu College of Science and Technology, Kamalpokhari, Kathmandu. During the study, 388 urine samples from suspected urinary tract infected cases were collected from both genders of children below 15 years of age from Out and In-patient department of International Children Friendship Hospital, Maharajgunj. For sample collection, In case of neonates and infants, genital area was first cleaned with sterile water and wiped from front to back until area is clean. For female, urine bag was affixed over genital area, starting from the perineum and working upwards. For male, urine bag was placed over the penis ensuring a tight seal all around the bag. Urine bag was checked frequently and removed as soon as the urine is passed. The parents of toilet trained children were suggested to collect midstream clean catch urine and were transported to our laboratory using ice box as soon as possible. However, improperly labeled, unlabeled and leaked sample were excluded from the study.

For processing of each sample, microbiological protocols were followed according to standard microbiological guidelines (Cheesbrough 2006 and Forbes et al. 2007). The collected urine samples were inoculated on MacConkey agar (MA) and Blood agar (BA) using a sterile calibrated loop. The inoculated plates were then incubated aerobically at $37^{\circ} \mathrm{C}$ for 24 hours. Colony count was made and plates showing more than or equal to $10^{5}$ colony forming units (cfu)/ $\mathrm{ml}$ of urine was considered for positive result (Forbes et al. 2007). The plates showing significant bacteriuria were then cultured for presumptive identification of $E$. coli and K. pneumoniae that was carried out on basis of colonial characteristics, gram staining and biochemical tests. 
The Kirby-Bauer disc diffusion method, according to the CLSI guidelines, was used to test the isolates for their antimicrobial susceptibilities using $\beta$-lactam antibiotics viz. cefpodoxime (30 $\mathrm{gg})$, cefotaxime $(30 \mu \mathrm{g})$, ceftrioxone $(30 \mu \mathrm{g}), \quad$ ceftazidime $(30 \mu \mathrm{g})$, cefepime $(30 \mu \mathrm{g})$, and non $\beta$-lactam antibiotics viz. gentamicin $(10 \mu \mathrm{g})$, ciprofloxacin $(5 \mu \mathrm{g})$, piperacillin/tazobactam $(100 / 10 \mu g)$, norfloxacin $(10 \mu \mathrm{g})$, amikacin $(30 \mu \mathrm{g})$, nitrofurantoin $(100 \mu \mathrm{g})$, cotrimoxazole $(25 \mu \mathrm{g})$, imipenem $(10 \mu \mathrm{g})$ and meropenem $(10 \mu \mathrm{g})$. All the antibiotic discs were procured from Hi-media, Mumbai (CLSI 2016).

In this study, if the isolates were resistant to at least one agent of three different classes of commonly used antimicrobial agents, they were regarded as multidrug resistant (MDR) (Magiorakos et al. 2012). If the zone of inhibition (ZOI) was $\leq 25 \mathrm{~mm}$ for Ceftriaxone, $\leq 22 \mathrm{~mm}$ for Ceftazidime, and/or $\leq 27 \mathrm{~mm}$ for Cefotaxime, the isolate was considered a potential ESBL producer as recommended by CLSI and further tested by confirmatory methods.

For Double disc synergy test, Mueller Hinton agar was inoculated with the standard ( $0.5 \mathrm{McF}$ arland) inoculum of the test isolate. Ceftazidime $(30 \mu \mathrm{g})$ disc was placed on agar $15 \mathrm{~mm}$ away from the center of amoxicillinclavulanic acid $(20 \mu \mathrm{g} / 10 \mu \mathrm{g})$ disc. Extension of zone of inhibition towards amoxicillin-clavulanic acid was interpreted as ESBL producer.

For phenotypic confirmatory test, disks of third generation cephalosporins alone and disks of third generation cephalosporins plus clavulanic acid are required for the phenotypic confirmatory test that uses combination disk method according to CLSI guidelines. Ceftazidime $(30 \mu \mathrm{g})$ disk alone and ceftazidime + clavulanic acid $(30 \mu \mathrm{g}+10 \mu \mathrm{g})$ disk; and cefotaxime $(30 \mu \mathrm{g})$ disk alone and cefotaxime + clavulanic acid $(30 \mu \mathrm{g}+10 \mu \mathrm{g})$ disk were used in this study. The disks were placed at a distance of at least $25 \mathrm{~mm}$ on a carpet culture of the isolate on MHA plate. Differences in zone diameters of cephalosporins alone and in combination with clavulanic acid were recorded after incubation for $16-18$ hours at $37^{\circ} \mathrm{C}$. The increase in zone diameter equal to or greater than $(\geq) 5 \mathrm{~mm}$ around cephalosporin plus clavulanic acid disk compared to cephalosporin alone indicates ESBL production by the organism.

The data of the case record forms were entered in the worksheet of Microsoft Excel. Frequency and percentages were analyzed as descriptive findings.

\section{RESULTS}

Out of a total of 388 mid-stream urine specimens (202 from male and 186 from female child) screened for significant bacteriuria, a total of 116 (29.89\%) were found to have significant growth from which bacterial isolates were obtained. Among total, 82/116 (70.68\%) and 18/116 (15.52\%) samples showed the growth of E. coli and K. pneumoniae, respectively. Rests of the bacterial isolates were Proteus vulgaris (4/116), Pseudomonas aeruginosa (2/116) and K. oxytoca (10/116). Initial screening of these isolates for ESBL production showed 42/82 of E. coli and 8/18 of K. pneumoniae strains to be ceftriaxone resistant. Confirmation test (PCDDT) revealed 34/82 (41.46\%) of E. coli and 8/18 $(44.44 \%)$ of K. pneumoniae isolates to be ESBL positive as shown in the following table.

Table 1: Distribution of bacterial isolates in urine sample

\begin{tabular}{lc}
\hline \multicolumn{1}{c}{ Bacterial isolates } & Number(\%) \\
\hline E. coli & $82(70.68)$ \\
K. pneumoniae & $18(15.52)$ \\
K. oxytoca & $10(8.62)$ \\
Proteus vulgaris & $4(3.45)$ \\
Pseudomonas aeruginosa & $2(1.73)$ \\
\hline Total & $\mathbf{1 1 6 ( 1 0 0 )}$ \\
\hline
\end{tabular}

Table 2: Phenotypic confirmation of ESBL producer from potentially screened isolates

\begin{tabular}{lcc}
\hline \multicolumn{1}{c}{ Isolates } & Total Screened for ESBL & Confirmed ESBL producers (\%) \\
\hline E. coli & 42 & $34(41.46)$ \\
K. pneumoniae & 8 & $8(44.44)$ \\
K. oxytoca & 2 & 0 \\
\hline Total & $52(44.82 \%)$ & $\mathbf{4 2}(36.20)$ \\
\hline
\end{tabular}


Among total ESBL isolates, highest rate of ESBL producers were from age group below 5 years $(66.66 \%)$ followed by age group 6-10 (23.80\%) and 11-15 (9.52\%).
ESBL production was more $(52.38 \%)$ from bacterial isolates of male child than female (47.62\%).

Table 3: Distribution of ESBL producers according to age and gender of children

\begin{tabular}{lccccccc}
\hline \multirow{2}{*}{ Age-Group (years) } & \multicolumn{2}{c}{ Gender } & \multirow{2}{*}{ Macterial isolates } & \multicolumn{2}{c}{ Gender } & \multirow{2}{*}{ ESBL producers (\%) } \\
\cline { 2 - 3 } \cline { 5 - 6 } & Male & Female & & & Male & Female & \\
\hline$\leq 5$ & 46 & 32 & 78 & 18 & 10 & $28(66.66)$ \\
$6-10$ & 16 & 8 & 24 & 4 & 6 & $10(23.80)$ \\
$11-15$ & 2 & 5 & 7 & 0 & 4 & $4(9.52)$ \\
\hline Total & $\mathbf{6 8}$ & $\mathbf{4 5}$ & $\mathbf{1 1 6}$ & $\mathbf{2 2 ( 5 2 . 3 8 )}$ & $\mathbf{2 0}(\mathbf{4 7 . 6 2 )}$ & $\mathbf{4 2}(\mathbf{1 0 0})$ \\
\hline
\end{tabular}

Among total bacterial isolates from Outpatient department, ESBL was produced from $46.87 \%$ isolates

whereas from inpatient isolates only $23.07 \%$ produce ESBL.

Table 4: Distribution of ESBL producers from patients attending different departments

\begin{tabular}{ccc}
\hline Patient department & Bacterial Isolates & ESBL producers(\%) \\
\hline Out-Patient & 64 & $30(46.87)$ \\
In-Patient & 52 & $12(23.07)$ \\
\hline Total & 116 & $\mathbf{4 2}$ \\
\hline
\end{tabular}

Among total MDR isolates, $67.74 \%$ produce ESBL and among MDR E. coli $73.91 \%$ produce ESBL and among

MDR K. pneumoniae, $80 \%$ produce ESBL whereas $K$. oxytoca did not produce ESBL.

Table 5: Distribution of ESBL producers among MDR isolates

\begin{tabular}{lcc}
\hline \multicolumn{1}{c}{ Bacterial isolates } & No. of MDR isolates (\%) & ESBL producer (\%) \\
\hline E. coli & $46(74.19)$ & $34(73.91)$ \\
K. pneumoniae & $10(16.13)$ & $8(80)$ \\
K. oxytoca & $6(9.68)$ & $0(0)$ \\
\hline Total & $\mathbf{6 2 ( 1 0 0 )}$ & $\mathbf{4 2 ( 6 7 . 7 4 )}$ \\
\hline
\end{tabular}

Among ESBL producing isolates, highest susceptibility was seen to Ceftazidime $(23.80 \%)$ followed by

Cefotaxime (16.67\%). The ESBL producing isolates were least susceptible to Ceftriaxone $(2.38 \%)$

Table 6: In vitro susceptibility of ESBL producers to $\beta$-lactam antibiotics

\begin{tabular}{lccc}
\hline \multicolumn{1}{c}{ Antibiotics } & E. coli $\mathbf{n}=\mathbf{3 4}$ & E. coli $\mathbf{n}=\mathbf{3 4}$ & Total $\mathbf{N}=\mathbf{4 2}$ Susceptible No. (\%) \\
\hline Ceftazidime & 8 & 8 & $10(23.80)$ \\
Ceftriaxone & 1 & 1 & $1(2.38)$ \\
Cefotaxime & 6 & 6 & $7(16.67)$ \\
Cefepime & 5 & 5 & $6(14.28)$ \\
Cefpodoxime & 4 & 4 & $5(11.90)$ \\
\hline
\end{tabular}

Upon testing of Susceptibility pattern for ESBL and non ESBL producers to non $\beta$-lactam antibiotics, a coresistance to the non- $\beta$ lactam antibiotics was observed more with the ESBL producers. Comparatively less resistance to amikacin and nitrofurantoin (19.1\% and 9.53\% respectively) was seen among ESBL producers. 40 out of 42 (95.23\%) ESBL producing strains showed susceptibility to piperacillin/tazobactam, a combination drug. The resistance to meropenem was observed to be less $(9.53 \%)$ as compared to that to imepenem $(7.15 \%)$ among ESBL producers while ESBL non-producers have shown absolute sensitivity towards meropenem, imipenem and piperacillin/tazobactam. 
Table 7: Antibiotic susceptibility of ESBL producers and non-producers towards non $\beta$-lactam antibiotics

\begin{tabular}{lcccc}
\hline \multirow{2}{*}{ Antibiotics } & \multicolumn{2}{c}{ ESBLproducers $(\mathbf{n = 4 2 )}$} & \multicolumn{2}{c}{ Non-producers $(\mathbf{n}=\mathbf{7 4 )}$} \\
\cline { 2 - 5 } & Susceptible No. (\%) & Resistant No. (\%) & Susceptible No. (\%) & Resistant No. (\%) \\
\hline Norfloxacin & $2(4.77)$ & $40(95.23)$ & $45(60.8)$ & $29(39.2)$ \\
Ciprofloxacin & $5(11.9)$ & $37(88.1)$ & $48(64.8)$ & $26(35.2)$ \\
Amikacin & $34(80.9)$ & $8(19.1)$ & $69(93.24)$ & $5(6.76)$ \\
Gentamicin & $11(26.19)$ & $31(73.81)$ & $65(87.83)$ & $9(12.17)$ \\
Co-trimoxazole & $8(19.04)$ & $34(80.96)$ & $33(44.59)$ & $41(55.41)$ \\
Nitrofurantoin & $38(90.47)$ & $4(9.53)$ & $70(94.59)$ & $4(5.41)$ \\
Piperacillin/Tazobactam & $40(95.2)$ & $2(4.8)$ & $74(100)$ & $0(0)$ \\
Meropenem & $38(90.47)$ & $4(9.53)$ & $74(100)$ & $0(0)$ \\
Imipenem & $39(92.85)$ & $3(7.15)$ & $74(100)$ & $0(0)$ \\
\hline
\end{tabular}

\section{DISCUSSION}

The occurrences of ESBLs among clinical isolates vary greatly worldwide and geographically and are rapidly changing overtime. The prevalence of ESBL producers was $36.20 \%$ (E.coli= $41.46 \%$ and K. pneumoniae $=$ $44.44 \%$ ) in this study. It correlates with a study done in India (Babypadmini and Appalaraju 2004) which reported nearly $40 \%$ of urinary isolates of E. coli and K. pneumoniae were ESBL positive. Findings from other studies in Nepal have shown ESBL production ranging from $18 \%$ to $62.7 \%$. (Shrestha et al. 2011; Poudyal et al. 2011; Thakur et al. 2013). Variation might have occurred due to low number of samples studied from different geographical locations. Similarly, variation in prevalence of ESBL producing organisms was found in other countries. Significant increase in ESBL organisms were published from India (Sasirekha et al. 2010 and Sharma et al. 2012), Pakistan (Ullah et al. 2009) and Nigeria (Yusha'u et al. 2010).

Previous studies from Nepal have reported the prevalence of the ESBL producing bacteria ranging from $13.5 \%$ to $33.2 \%$. Chander and Shrestha (2013) reported the ESBL prevalence rate to be $13.5 \%$ whereas, recently, Neupane et al. (2016) have reported 33.2\% of ESBL producers in their study. Similarly, Ansari et al. (2015), Khanal et al. (2013), have reported 24\% and 25\% ESBL producers, respectively. These findings show less prevalence with those of our study. However, extremely lower rates of ESBL production have been documented from Japan, Korea, and United States (Paterson and Bonomo 2005; Yan et al. 2014). The differences in the ESBL rates may be attributable to the geographic difference, antimicrobial stewardship programme, and infection control practices.

In this study, ESBL production was more $(52.38 \%)$ from isolates of male child than female (47.62\%).
However, Females showed a higher rate of isolation of ESBL producing E. coli $(60 \%)$ and K. pneumoniae $(62.5 \%)$, which discords the findings as reported earlier (Oladeinde et al. 2011 and Ahmed et al. 2012 ). The result is inconsistent because ESBL production and pathogenic nature may not differ according to gender. However, in our country, preference is given to male child and parents take care of them with so much love and take them to hospital in a higher frequency. This may be the reason behind higher inclusion of male child in this study.

The highest bacterial isolates were found in children less than 5 years age, including the prevalence of ESBL organisms which was above $66.66 \%$. The reason for this may be due to the immunological status of the children below 5 years of age who are more vulnerable to infections, malnourished child, and child living in poor sanitation. Similar result was observed in a study done by Kayastha et al. (2020) in Nepal. The higher prevalence of bacterial growth in outpatients, in this study indicates the emergence of ESBL producing pathogens from community that may spread creating difficulties in treating the patients with drug resistance. However, this result is contrary to a study in Nepal (Kayastha et al. 2020) which showed greater prevalence in inpatients which may have been added by nosocomial infections associated with prolonged hospital stay, intensive care unit admission, extensive use of invasive medical devices, and overconsumption of antibiotic among inpatients.

MDR was found in $53.44 \%(62 / 116)$ of the urinary isolates, among them, major MDR producer was E. coli. Among total MDR isolates, $67.74 \%$ produce ESBL and among MDR E. coli $73.91 \%$ produce ESBL and among MDR K. pneumoniae, $80 \%$ produce ESBL. Production of different $\beta$-lactamase, hydrolyze $\beta$-lactam ring of 
antibiotic, like TEM-1, TEM-2, SHV-1 and many other plasmid-mediated $\beta$-lactamases confers high level of resistance to drug in E. coli. Furthermore, different efflux pumps and target site mutation at gyrA and parC are responsible for fluoroquinolones resistance (Sharma et al. 2018).

Among ESBL producing isolates, highest susceptibility was seen to Ceftazidime $(23.80 \%)$ followed by Cefotaxime (16.67\%). The ESBL producing isolates were least susceptible to Ceftriaxone (2.38\%). The high rates for non-ESBL mediated ceftriaxone resistant isolates may be due to their different mechanisms for resistance such as the production of ampC $\beta$ lactamase, metallo-betalactamase, etc. (Dalela et al. 2012). This further limits the therapeutic options available to treat these infections. In our study, false susceptibilities to ceftazidime and cefotaxime were observed in $23.8 \%$ and $16.67 \%$ of the ESBL producers. This could be due to the reason that the optimal substrate profile varies from one ESBL enzyme to another (Wong 2001). Hence, the susceptibility panels with only one extended spectrum cephalosporin cannot predict the resistance to the other extended spectrum cephalosporins (Rice and Jao 1991).

Due to the difficulty in detection of ESBL by the current clinical methods, many of these strains have been falsely reported to be susceptible to the widely used broad-spectrum $\beta$-lactams, ESBLs constitute a serious threat to the $\beta$-lactam therapy (Mackenzi et al. 2002). The ESBL producers are intrinsically resistant to all the cephalosporins even if they exhibit an in vitro susceptibility. The ESBL production coexists with the resistance to several other antibiotics.

In the study, upon testing the susceptibility pattern for ESBL and non-ESBL producers to non $\beta$-lactam antibiotics, a co-resistance to the non $\beta$-lactam antibiotics was observed more with the ESBL producers. Comparatively less resistance to amikacin and nitrofurantoin (19.1\% and 9.53\% respectively) was seen among ESBL producers. In the study, 95.23\% ESBL producing strains showed susceptibility to the combination drug, piperacillin/tazobactam. The resistance to meropenem was observed to be less $(9.53 \%)$ as compared to that to imepenem (7.15\%).

Aco-resistancetothequinolonesand theaminoglycosides is common. We found such an associated resistance of ESBL producers to co-trimoxazole (80.96\%), gentamicin (73.81\%) and the flouroquinolones (88.10-95.23\%).
Another study reported 91.17\%, 100\% and 94.91\% resistances respectively to gentamicin, cotrimoxazole and ciprofloxacin in the ESBL producers (Gupta et al. 2007). The high resistance to the non $\beta$-lactam antibiotics of the ESBL producing strains poses a threat of treatment failure by these drugs and it also minimizes the therapeutic choice to the carbapenems. Hence, the emerging resistance to the carbepenems is a phenomenon of great concern for combating the infections of the multidrug resistant bacteria (Parveen et al. 2010). Although $\beta$ - lactam/ $\beta$ - lactamase inhibitor combinations have been suggested as the treatment option for ESBL producers, these drugs must be given in high doses in lower frequency, so that serum and tissue levels of these combinations are higher with a correspondingly higher clinical success rate. (Adam 2002).

\section{CONCLUSION}

This study concluded that there is high prevalence of multidrug resistant uropathogenic clinical strains of E. coli and K. pneumoniae with higher rates of ESBL production. These strains show lower rate of sensitivity to $\beta$-lactam antibiotics even they are capable of producing ESBL and higher rate of sensitivity to combination therapy. A resistance to the carbepenems is also emerging. Appropriate antimicrobial regimen selection for empirical therapy is thus important for such cases. On managing the empirical antibiotics practice, one can reduce the risk of ESBL producers. There is an essence need of regular routine practice of ESBL detection.

\section{ACKNOWLEDGEMENTS}

We would like to thank all the patients whose samples are tested in the study, all laboratory staff, and faculties of KCST, our friends and relatives who directly and indirectly supported us for the study.

\section{CONFLICT OF INTEREST}

The authors declare no conflict of interest.

\section{REFERENCES}

Adam D (2002) Beta-lactam/Beta-lactamase Inhibitor Combinations in Empiric Management of Pediatric Infections. J Int Med Res 30: 10-19.

Ahmed SM, Jakribettu RP, Koyakutty S, Arya B and Shakir VPA (2012) Urinary tract infections - an overview on the prevalence and the anti-biogram of gram negative uropathogens in a tertiary care center in North Kerala, India. J Clin Diagn Res 
6:1192-1195.

Ansari S, Nepal HP, Gautam R, Shrestha S, Neopane P, Gurung G and Chapagain ML (2015) Community acquired multi-drug resistant clinical isolates of Escherichia coli in a tertiary care center of Nepal. Antimicrob Resist Infect Control 4:15.

Babypadmini S and Appalaraju B (2004) Extended spectrum lactamase in urinary isolates of E.coli and Klebsiella pneumoniae - prevalence and susceptibility patterns in a tertiary care hospital. Indian J Med Microbiol 22:172-174.

Briongos-Figuero LS, Gomez-Traveso T, BachillerLuque P, Dominguez-Gil Gonzalez M, GomezNieto A, Palacios-Martin T, González-Sagrado M, Dueñas-Laita A, Pérez-Castrillón JL (2012). Epidemiology, risk factors and comorbidity for urinary tract infections caused by extendedspectrum beta-lactamase (ESBL)-producing enterobacteria. Int J Clin Pract 66:891-6.

Chander A and Shrestha CD (2013) Prevalence of extended spectrumbeta lactamase producing Escherichia coli and Klebsiella pneumoniae urinary isolates in a tertiary care hospital in BioMed Research International 7 Kathmandu, Nepal. BMC Research Notes 6(1):487.

Cheesbrough M (2006) District Laboratory Practice in Tropical Countries. 2nd edition: Cambridge University Press, Cambridge, UK.

Clinical and Laboratory Standards Institute (CLSI) Performance Standards for Antimicrobial Susceptibility Testing (M100S). Wayne, PA: CLSI; 2016.

Coque TM, Baquero F and Canton R (2008) Increasing prevalence of ESBL producing enterobacteriaceae in Europe. Eurosurveillance 13(47):1-11.

Dalela G, Gupta S, Jain DK and Mehta P (2012) Antibiotic resistance patterns in uropathogens at a tertiary care hospital at Jhalawar with special reference to ESBL, AmpC b-lactamase and MRSA production. J Clin Diagn Res 6(4):645-651.

Forbes BA, Sahm FD and Weissfelt SA (2007) Bailey and Scott's diagnostic Microbiology. Mosby Publication.

Ghedira L, Messaoudi A, Ben MC and Guediche MN (2004) Profile of antimicrobial resistance of agents causing urinary tract infections in children. Tunis Med 82.

Gupta V, Singla N and Chander J(2007) Detection of
ESBLs using third and fourth cephalosporins in double disc synergy test. Indian J Med Res 126:48687.

Hammer DA, Dongol S, Anderson TP, Wong JS, Werno AM and Murdoch DR (2007) High prevalence of extended-spectrum beta-lactamase-producing Enterobacteriaceae in Nepal. Int J Antimicrob Agents 30(5): 471-2.

Hoban DJ, Nicolle LE, Hawser S, Bouchillon S and Badal R (2011) Antimicrobial susceptibility of global inpatient urinary tract isolates of Escherichia coli: Results from the Study for Monitoring Antimicrobial Resistance Trends (SMART) program: 2009-2010. Diagn Microbiol Infect Dis. 70:507-11.

Kariuki S, Revathi G, Corkill J, Kiiru J, Mwituria J, Mirza N and Anthony CH (2007) Escherichia coli from community-acquired urinary tract infections resistant to fluoroquinolones and extended-spectrum beta-lactams. I Infect Dev Ctries 1:257-62.

Kayastha K, Dhungel B, Karki S, Adhikari B, Banjara M R, Rijal K R and Ghimire P (2020) ExtendedSpectrum $\beta$-Lactamase-Producing Escherichia coli and Klebsiella species in Pediatric Patients Visiting International Friendship Children's Hospital, Kathmandu, Nepal. Infectious Diseases: Research and Treatment 13: 1-7.

Khanal S, Joshi DR, Bhatta DR, Devkota U and Pokhrel BM (2013) $\beta$-lactamase-producing multidrugresistant bacterial pathogens from tracheal aspirates of intensive care unit patients at national institute of neurological and allied sciences, Nepal. ISRN Microbiology 2013:5.

Livermore DM, Canton R, Gniadkowski M, Nordmann P, Rossolini GM, Arlet G, Ayala J, Coque TM, Kern-Zdanowicz I, Luzzaro F, Poirel L and Woodford N (2007) CTX-M: changing the face of ESBLs in Europe. J Antimicrob Chemother 59(2): 165-174.

MacKenzie FM, Miller FM CA and Gould IM (2002) Comparison of screening methods for TEM and SHV derived ESBL detection. Clin Microbiol Infect 8:715-24

Magiorakos AP, Srinivasan A, Carey RB, Carmeli Y, Falagas ME, Giske CG, Harbarth S, Hindler JF, Kahlmeter G, Olsson-Liljequist B, Paterson DL, Rice LB, Stelling J, Struelens MJ, Vatopoulos A, Weber JT and Monnet DL (2012) Multidrug- 
resistant, extensively drug-resistant and pandrugresistant bacteria: an international expert proposal for interim standard definitions for acquired resistance Clin Microbiol Infect. 18(3): 268-81.

Neupane S, Pant ND, Khatiwada S, Chaudhary R and Banjara MR (2016) Correlation between biofilm formation and resistance toward different commonly used antibiotics along with extended spectrum beta lactamase production in uropathogenic Escherichia coli isolated from the patients suspected of urinary tract infections visiting Shree Birendra Hospital, Chhauni, Kathmandu, Nepal. Antimicrob Resist Infect Control 5: 5 .

Oladeinde BH, Omoregie R, Olley $\mathrm{M}$ and Anunibe JE (2011) Urinary tract infections in a rural community of Nigeria. North Amer J Med Sci 3:7577.

Parveen RM, Harish BN and Parija SC (2010) Emerging carbepenem resistance among nosocomial isolates of Klebsiella pneumoniae in South India. Inter J Pharm Bio Sci 1(2): 1-11.

Paterson DL and Bonomo RA (2005) Extendedspectrum $\beta$ - lactamases: a clinical update.Clinical Microbiology Reviews 18 (4): 657-686.

Poudyal S, Bhatta DR, Shakya G, Upadhyaya B, Dumre SP, Buda G and Kandel BP (2011) ESBL producing multi drug resistant clinical bacterial isolates at NPHL, Nepal. Nepal Med Coll J 13: 34-38.

Rice LB and Jao JD (1991) Efficacy of different $\beta$-lactams against an ESBL producing Klebsiella pneumoniae strains in the rat intra abdominal abscess model. Antimicrob Agents Chemther 35: 1243-44.

Sasirekha B, Manasa R, Ramya P and Sneha R (2010) Frequency and Antimicrobial Sensitivity Pattern Of Extended Spectrum $\beta$-Lactamases Producing E. coli and K. pneumoniae Isolated In A Tertiary Care Hospital. Al Ameen J Med Sci 3: 265- 271.

Schwaber MJ and Carmeli Y (2007) Mortality and delay in effective therapy associated with extended-spectrum $\beta$-lactamase production in Enterobacteriaceae bacteraemia: a systematic review andmeta-analysis. I Antimicrob Chemother 60(5): 913-920.

Sharma KR, Bhandari P, Adhikari N, Tripathi P, Khanal S and Tiwari BR (2018) Extended Spectrum $\beta$-lactamase (ESBL) Producing Multi
Drug Resistant (MDR) Urinary Pathogens in a Children Hospital from Nepal. Kathmandu Univ Med J 62(2): 151-5.

Sharma S, Gupta A and Arora A (2012) Cefepime Tazobactam: A new $\beta$-lactam/ $\beta$-lactamase inhibitor combination against ESBL producing gram negative bacilli. Int J Pharm Biomed Sci 3: 3538.

Shrestha S, Amatya R and Dutta R (2011) Prevalence of extended spectrum $\beta$-lactamase (ESBL) production in gram negative isolates from pyogenic infection in tertiary care hospital of eastern Nepal. Nepal Med Coll J 13:186-189.

Talbot GH, Bradley J, Edwards JE, Jr., Gilbert D, Scheld $\mathrm{M}$ and Bartlett JG (2006) Bad bugs need drugs: an update on the development pipeline from the Antimicrobial Availability Task Force of the Infectious Diseases Society of America. Clin Infect Dis. 42:657-68.

Thakur S, Pokhrel N and Sharma M (2013) Prevalence of Multidrug Resistant Enterobacteriaceae and Extended Spectrum $\beta$-Lactamase Producing E. coli in Urinary Tract Infection. Res J Pharm Biol Chem Sci 4:1615-1624.

Ullah F, Malik SA and Ahmed J (2009) Antibiotic susceptibility pattern and ESBL prevalence in nosocomial Escherichia coli from urinary tract infections in Pakistan. Afr J Biotechnol 8(16):39213926.

Ullah F, Malik SA and Ahmed J (2009) Antimicrobial susceptibility pattern and ESBL prevalence in K. pneumoniae from urinary tract infections in the North West of Pakistan. Afr J Microbiol Res 3:676680 .

Wong BA (2001) Therapeutic challenges associated with extended spectrum $\beta$-lactamase producing E. coli and Klebsiella pneumoniae. Pharmacotherapy 21: 583-92.

Yan YZ, Sun KD, Pan LH, Fan HQ, Yang HZ, Lu YC and Shi Y (2014) A screening strategy for phenotypic detection of carbapenemase in the clinical laboratory. Can J Microbiol 60(4): 211-215.

Yusha'u M, Aliyu HM, Kumurya AS and Suleiman K (2010) Prevalence of extended spectrum $\beta$-lactamases (ESBLs) among Enterobacteriaceae in Murtala Mohammed Specialist Hospital, Kano, Nigeria. Bayero J Pure Appl Sci 3(1): 169-172. 